\title{
VA Announces Aggressive New Approach to Produce Rapid Improvements in VA Medical Centers
}

The U.S. Department of Veterans Affairs (VA) announced steps that it is taking as part of an aggressive new approach to produce rapid improvements at VA's low-performing medical facilities nationwide (1). VA defines its low-performing facilities as those medical centers that receive the lowest score in its Strategic Analytics for Improvement and Learning (SAIL) star rating system, or a one-star rating out of five. The SAIL star rating was initiated in 2016 and uses a variety of measures including mortality, length of hospital stay, readmission rates, hospital complications, physician productivity and efficiency. A complete listing of the VA facilities, their star ratings and the metrics used to determine the ratings is available through the end of fiscal year 2017 (2). Based on the latest ratings, the VA currently has 15 one-star facilities including Denver, Loma Linda, and Phoenix in the Southwest (Table 1).

Table 1. VA facilities with one-star ratings (1).

1. Big Spring (Texas)

2. Denver (Colorado)

3. Dublin (Georgia)

4. El Paso (Texas)

5. Jackson, (Mississippi)

6. Hampton (Virginia)

7. Harlingen (Texas)

8. Loma Linda (California)

9. Memphis (Tennessee)

10. Murfreesboro (Tennessee)

11. Nashville (Tennessee)

12. Phoenix (Arizona)

13. Roseburg (Oregon)

14. Walla Walla (Washington).

15. Washington (DC)

The steps VA is taking to produce rapid improvements at its low-performing facilities include (Table 2):

Table 2. VA steps to produce rapid improvements at low-performing facilities (1).

1. Central, national accountable leadership - VA has designated Dr. Peter Almenoff, Director of VA's Office of Reporting, Analytics, Performance, Improvement and Deployment (RAPID) Healthcare Improvement Center, to oversee improvement at each of the centers.

2. Comprehensive analysis and identification of improvement targets VA is employing a new initiative, known as Strategic Action Transformation (STAT), that uses a rigorous and formal approach based 
on clinical performance indicators to identify vulnerabilities in each lowperforming facility and set specific targets for improvement.

3. Provision of national resources for improvement - VA's RAPID team of experts will use sophisticated statistical tools to track the progress of improvement against these targets, and, where warranted, will dispatch a team of expert improvement coaches quickly to the medical centers to assist them in meeting the goals.

4. Accountability for results -VA's Central Office will review each of the facilities quarterly, and if the facilities fail to make rapid substantial progress in their improvement plan, VA leadership will take prompt action, including changing the leadership of the medical center.

VA secretary David Shulkin stated that "President Trump has made it clear that our Veterans deserve only the best when it comes to their healthcare, and that's why we are focusing on improving our lowest performing facilities nationwide" (1). The VA recently removed the Roseburg Oregon VA Medical Center director who was accused of manipulating hospital admissions to improve the hospital's rating (3). Almenoff, the overseer of improvement, was transferred from his position as the VA Integrated Network 15 director in 2008 when the Marion VA came under fire for substandard care raising concerns from several Illinois legislators, including the then junior senator from Illinois, Barack Obama (4).

A major hurdle will be for the VA to hire sufficient staff to improve care. As of the end of June, the VA reported 35,554 job vacancies system-wide, and VA Secretary David Shulkin has cited challenges with hiring doctors and nurses, particularly mental health care professionals (5). The agency set a goal to hire 1,000 mental health care workers in 2017. The VA hired 900 last year, but lost 945. The Veterans Access, Choice and Accountability Act of 2014 appropriated several billion dollars to the VA but this apparently did not lead to hiring of sufficient healthcare providers.

Richard A. Robbins, MD

Editor, SWJPCC

\section{References}

1. VA Office of Public and Intergovernmental Affairs. VA announces aggressive new approach for low-performing medical centers. February 1, 2018.

Available at: https://www.va.gov/opa/pressrel/pressrelease.cfm?id=4004 (accessed 2/14/18).

2. US Department of Veterans Affairs. Quality care. Available at: https://www.va.gov/QUALITYOFCARE/measureup/Strategic Analytics for Improvement and Learning SAIL.asp (accessed 2/14/18).

3. Phillips D. Director of veterans hospital accused of manipulating ratings is replaced. New York Times. February 1, 2018. Available at: 
https://www.nytimes.com/2018/02/01/us/veterans-roseburg-director.html (accessed 2/14/18).

4. Durbin D, Obama B, Costello J, Shimkus J. Letter to The Honorable James B. Peake, M.D., Secretary of Veterans' Affairs. July 23, 2008. Available at: https://votesmart.org/public-statement/363179/letter-to-the-honorable-jamesb-peake-md-secretary-of-veteransaffairs?flavour=mobile\&utm source=votesmart\&utm medium=mobilelink\&utm campaign=flavourswitch\#.WoR3Dq6nGUk (accessed 2/14/18).

5. Wentling N. Federal unions march on VA headquarters to protest staffing shortages. Stars and Stripes. February 13, 2018. Available at: https://www.stripes.com/federal-unions-march-on-va-headquarters-to-proteststaffing-shortages-1.511543 (accessed 2/14/18). 